\title{
Receding-Horizon Model Predictive Control for a Three-Phase VSI with an LCL Filter
}

\author{
Ramon Guzman, Luis Garcia de Vicuña, Antonio Camacho, Jaume Miret,Member,IEEE, Juan M. Rey
}

\begin{abstract}
This paper presents a Continuous Control Set Model Predictive Control with a receding horizon for a three-phase voltage source inverter with an LCL filter. In this proposal, a reduced model of the converter with an embedded integrator and a Kalman filter are used to obtain the inverter-side currents without oscillation. Then, a cost function makes use of these currents to generate the optimum duty cycles for the space vector modulator. With the proposed method, active damping performances, a zero state error and a reduction of the computational burden are achieved. Compared to the Finite Control Set Model Predictive Control, the proposed method operates with a fixed switching frequency. Simulations and experimental results show that this proposal works correctly even in the case of grid harmonics and voltage sags.
\end{abstract}

Index Terms-Current control, LCL filter, Model predictive control, Kalman filter.

\section{INTRODUCTION}

$\mathbf{T}$ HE energy sector is increasingly leaning toward smart grids (SGs), thus maximizing the penetration of distributed generation (DG) systems [1], [2]. Most of these DG units are interfaced to the utility grid by means of a voltage source inverter (VSI) [3] connected through an LCL filter. This option allows a better attenuation of switching harmonics but leads to an inherent resonance problem which can be overcome using either passive or active damping techniques. The former is the simplest solution. It involves including a physical resistor in series with the filter capacitor. However, this option causes system losses which may be unacceptable in some applications, for instance in wind turbines, since they operate typically at $30 \%$ of the converter nominal power [4]. On the contrary, active damping techniques can be considered.

Manuscript received March 26, 2018; revised June 8, 2018 and August 9, 2018; accepted October 9, 2018.

This work has been supported by the European Union ProjectELAC2014/ESE0034 and its linked to Spanish National Project PCIN-2015-001 and also supported by the Ministry of Economy and Competitiveness of Spain under project ENE2015-64087-C2-1-R.

Ramon Guzman and Antonio Camacho are with the Department of Automatic control, Technical University of Catalonia, 08800 Vilanova i la Geltru, Spain (email:ramon.guzman@upc.edu;camachem@gmail.com).

J.M. Rey is with the Escuela de Ingenieras Elctrica, Electrnica y de Telecomunicaciones, Universidad Industrial de Santander, Bucaramanga 680002, Colombia, and also with the Department of Electronic Engineering, Universitat Politecnica de Catalunya, Vilanova i la Geltru 08800, Spain (e-mail: juanmrey@uis.edu.co).

Other authors are with the Department of Electronic and Electrical Engineering, Technical University of Catalonia, 08800 Vilanova i la Geltru, Spain(e-mail:vicuna@eel.upc.edu; jmiret@eel.upc.edu).
In this case, the system losses can be reduced, since the damping is obtained by modifying the control algorithm, and no physical resistors are needed [5], [6].

In this way, designing proper controllers has been the main interest of researchers. Different techniques for controlling VSIs have been presented in the literature, such as the sliding mode control (SMC) [7], [8], the optimal control [9] or the model predictive control (MPC) [10]-[12], among others. With the development of digital signal processors (DSPs), the MPC has become a promising control method due to its advantages, such as a fast tracking response, a high control bandwidth and providing a very simple way of including system nonlinearities and constraints [13], [14].

Two different control strategies regarding the MPC algorithms can be found in the literature: the Finite Control Set Model Predictive Control (FCS-MPC) [15]-[17] and the Continuous Control Set Model Predictive Control (CCS-MPC) [18]-[20]. In the former, the optimization problem is reduced to a finite number of switching states. In this technique, a cost function is calculated for every switching state, each one related to a specific voltage vector. Then, the switching state which minimizes the error between the current and its reference is applied to the converter. Unlike other control techniques, such as Proportional Integral (PI) or Proportional Resonant (PRES) controllers, this control method involves a direct drive of inverter switches without the use of PWMbased techniques. An interesting characteristic of this control strategy is its very fast transient response and the large control bandwidth. Another attractive performance is the possibility of including some restrictions due to the flexible nature of the cost function. However, the non-desirable variable switching frequency can be considered as the main drawback of this approach [13].

As an alternative, and with the aim of overcoming such an inconvenience, the CCS-MPC technique can be adopted. In this method, the control algorithm is based on the prediction of the state variables according to a discrete model of the power converter. These state variables can be estimated using a conventional state observer, but other estimation strategies can be adopted. [8]. In any case, the predicted state variables are used in a cost function, which is evaluated over a prediction horizon in order to obtain the vector of future control actions. If a receding horizon is considered, only the first value of this vector is used in the control algorithm. Unlike the FCSMPC, a continuous duty cycle is obtained which allows using a space vector modulator (SVM). Compared to the FCS-MPC, this approach has several advantages, such as an improvement 
of the total harmonic distortion (THD) of the three-phase currents and a fixed switching frequency [21]. It is important to remark that control signals can be directly applied to the SVM without the use of PI or PRES controllers, avoiding the detuning problems associated with this kind of controllers [22].

This paper proposes a CCS-MPC with a receding-horizon for a VSI with an LCL filter. A modified model of the converter with a reduced number of state variables is considered. Besides, an embedded integrator is added to the model in order to eliminate model uncertainties, and also to achieve a zero steady state error. The model is also used in a Kalman filter (KF) to estimate three-phase grid currents without oscillation, thus allowing active damping. Note that the use of a KF instead of a conventional state observer can be advantageous in noisy environments [8], also helping to reduce the switching noise. Finally, a feedforward term is added to generate the control signals with the aim of compensating the non-desirable grid harmonics, without using any harmonics model or any direct control of the grid currents [23].

As far as authors know, the CCS-MPC had not been applied for this purpose until now due to the large number of operations involved in this technique [13]. The results show that it is possible to implement the CCS-MPC in a conventional DSP.

The main advantages of this proposal can be summarized as follows:

- The use of the reduced model in the CCS-MPC allows active damping and a reduction of the computational burden.

- An embedded integrator is used in this model in order to eliminate model uncertainties and also to achieve a zero steady state error.

- A fixed switching frequency is achieved.

- The THD of the grid currents can be reduced in a significant way, compared to the FCS-MPC.

- A feedforward term can be easily introduced to minimize the harmonic content of grid currents.

This paper is organized as follows. In Section II a bilinear model of the VSI with an LCL filter is presented. Section III presents the proposed control system. In section IV, a method to compensate grid harmonics is proposed, and in section $\mathrm{V}$ a positive sequence extractor is presented. The implementation of the controller is presented in section VI, and experimental results are reported in section VII. A comparative analysis between the FCS-MPC and the CCS-MPC is presented in section VIII. Finally, section IX draws some conclusions of this proposal.

\section{Physical Model equations for a threE-Phase GRID-CONNECTED VSI WITH AN LCL FILTER}

Fig. 1 shows a three-phase grid-connected VSI with an LCL filter, where the grid impedance is considered pure inductive.

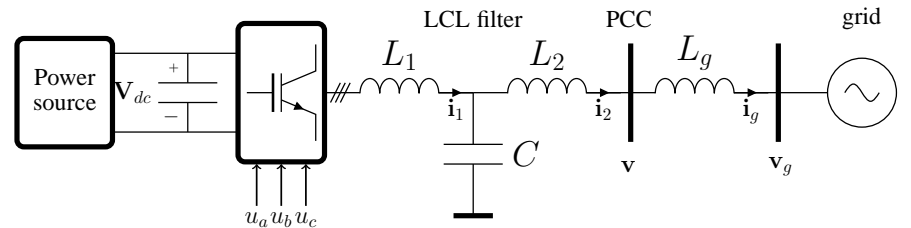

Fig. 1. Circuit diagram of a three-phase grid-connected inverter with an LCL filter

The VSI equations in the $\alpha \beta$ frame can be expressed as follows:

$$
\begin{aligned}
L_{1} \frac{d \mathbf{i}_{1 \alpha \beta}}{d t} & =\frac{V_{d c}}{2} \mathbf{d}_{\alpha \beta}-\mathbf{v}_{c \alpha \beta} \\
C \frac{d \mathbf{v}_{c \alpha \beta}}{d t} & =\mathbf{i}_{1 \alpha \beta}-\mathbf{i}_{2 \alpha \beta} \\
\left(L_{2}+L_{g}\right) \frac{d \mathbf{i}_{2 \alpha \beta}}{d t} & =\mathbf{v}_{c \alpha \beta}-\mathbf{v}_{g \alpha \beta}
\end{aligned}
$$

where $\mathbf{i}_{1 \alpha \beta}=\left[\begin{array}{ll}i_{1 \alpha} & i_{1 \beta}\end{array}\right], \mathbf{i}_{2 \alpha \beta}=\left[\begin{array}{ll}i_{2 \alpha} & i_{2 \beta}\end{array}\right], \mathbf{v}_{c \alpha \beta}=\left[\begin{array}{ll}v_{c \alpha} & v_{c \beta}\end{array}\right]$, $\mathbf{v}_{g \alpha \beta}=\left[\begin{array}{ll}v_{g \alpha} & v_{g \beta}\end{array}\right]$, and $\mathbf{d}_{\alpha \beta}=\left[\begin{array}{ll}d_{\alpha} & d_{\beta}\end{array}\right]$ are the vectors of the inverter-side currents, the grid-side currents, the capacitor voltages, the grid voltages and the control signals in the $\alpha \beta$ frame, respectively.

\section{Proposed Control System}

The proposed control system is shown in Fig.2. In this control scheme, the three-phase voltages and currents are transformed into the $\alpha \beta$ coordinates and used in a KF estimator to predict the inverter-side currents and the voltages at the point of common coupling (PCC). On one hand, the predicted currents will be used in a cost function in order to obtain the optimum control signals. On the other hand, the estimated PCC voltages and their quadratures will allow us to determine the positive sequence of the grid voltages. From the positive sequence and the desired active and reactive powers, reference currents will be generated. Note that in the case of voltage sags, only the positive sequence of voltages will be considered in order to achieve balanced reference currents. Finally, in the case of grid harmonics, a feedforward term is introduced to reduce the THD of grid currents. That will require that the measured voltages in the $\alpha \beta$ frame are scaled by a factor $2 / V_{d c}$ and added to the signals $u_{\alpha}$ and $u_{\beta}$ obtained from the cost function minimization. Finally, the resulting duty cycles, $d_{\alpha}$ and $d_{\beta}$ are applied to an SVM, allowing to fix the switching frequency of the VSI. In section IV a clear explanation of this procedure will be presented.

\section{A. Proposed discrete model with embedded integrator}

In order to reduce the control algorithm computational burden and also to achieve active damping performances, a reduced model of the converter will be used [7]. The proposed model only considers a single equivalent inductor and neglects the filter capacitor effect of the LCL tank. Then, according to 


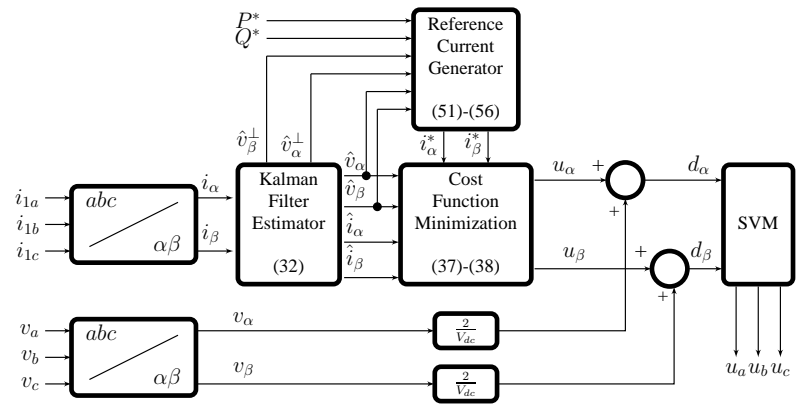

Fig. 2. Proposed control system

[7], the reduced model of the VSI in the $\alpha \beta$ frame can be represented as follows:

$$
\begin{aligned}
\left(L_{1 o}+L_{2 o}\right) \frac{d \mathbf{i}_{1 \alpha \beta}}{d t} & =\frac{V_{d c o}}{2} \mathbf{d}_{\alpha \beta}-\mathbf{v}_{\alpha \beta} \\
\frac{d \mathbf{v}_{\alpha \beta}}{d t} & =\omega_{o} \mathbf{v}_{\alpha \beta}^{\perp} \\
\frac{d \mathbf{v}_{\alpha \beta}^{\perp}}{d t} & =-\omega_{o} \mathbf{v}_{\alpha \beta}
\end{aligned}
$$

where $L_{1 o}$ and $L_{2 o}$ are the nominal values of the input and output inductors, $\omega_{o}$ is the grid angular frequency, $V_{d c o}$ is the nominal dc-link voltage, and $\mathbf{v}_{\alpha \beta}^{\perp}=\left[\begin{array}{lll}v_{\alpha}^{\perp} & v_{\beta}^{\perp}\end{array}\right]$ is the quadrature voltage at the PCC.

Since a digital implementation is required, the proposed model must be discretized with a sampling period $T_{s}$. From (4)-(6), the reduced discrete state space model for both $\alpha$ and $\beta$ channels can be represented as follows:

$$
\begin{aligned}
\mathbf{x}_{m, i}(k+1) & =\mathbf{A}_{m} \mathbf{x}_{m, i}(k)+\mathbf{B}_{m} u_{i}(k)+\boldsymbol{\eta}_{i} \\
y_{i}(k) & =\mathbf{C}_{m} \mathbf{x}_{m, i}(k)
\end{aligned}
$$

where $i \in\{\alpha, \beta\}$ and

$$
\begin{gathered}
\mathbf{A}_{m}=\left(\begin{array}{ccc}
1 & -T_{s} /\left(L_{1 o}+L_{2 o}\right) & 0 \\
0 & 1 & T_{s} \omega_{o} \\
0 & -T_{s} \omega_{o} & 1
\end{array}\right) \\
\mathbf{B}_{m}=\left(\begin{array}{ccc}
\frac{V_{d c o} T_{s}}{2\left(L_{1 o}+L_{2 o}\right)} & 0 & 0
\end{array}\right)^{T} \\
\mathbf{C}_{m}=\left(\begin{array}{lll}
1 & 0 & 0
\end{array}\right)
\end{gathered}
$$

being the state-space vector defined as:

$$
\mathbf{x}_{m, i}(k)=\left(\begin{array}{lll}
i_{1 i} & v_{i} & v_{i}^{\perp}
\end{array}\right)^{T}
$$

In (7), $\boldsymbol{\eta}_{i}$ represents a constant vector which contains the steady-state parameters and the model uncertainties and regarded as an input disturbance.

On the other hand, in a similar way as described in [14], an integrator can be embedded in the aforementioned statespace model with the aim of ensuring a zero steady-state error. Then, by considering both sides of (7), through the difference operation, we obtain:

$$
\begin{aligned}
& \mathbf{x}_{m, i}(k+1)-\mathbf{x}_{m, i}(k)=\mathbf{A}_{m}\left(\mathbf{x}_{m, i}(k)-\mathbf{x}_{m, i}(k-1)\right) \\
& +\mathbf{B}_{m, i}\left(u_{i}(k)-u_{i}(k-1)\right)+\boldsymbol{\eta}_{i}-\boldsymbol{\eta}_{i} .
\end{aligned}
$$

or equivalently

$$
\Delta \mathbf{x}_{m, i}(k+1)=\mathbf{A}_{m} \Delta \mathbf{x}_{m, i}(k)+\mathbf{B}_{m} \Delta u_{i}(k)
$$

In a similar way, the output variable can be determined in its incremental dynamics as follows:

$$
\begin{aligned}
y_{i}(k+1)-y_{i}(k) & =\mathbf{C}_{m}\left(\mathbf{x}_{m, i}(k+1)-\mathbf{x}_{m, i}(k)\right) \\
& =\mathbf{C}_{m} \mathbf{A}_{m} \Delta \mathbf{x}_{m, i}(k)+\mathbf{C}_{m} \mathbf{B}_{m} \Delta u_{i}(k)
\end{aligned}
$$

Now, a new the state-space vector can be defined, which contains the vectors $\Delta \mathbf{x}_{m, i}(k)$ and $y(k)$, yielding:

$$
\mathbf{x}_{i}(k)=\left[\begin{array}{ll}
\Delta \mathbf{x}_{m, i}(k) & y_{i}(k)
\end{array}\right]^{T}
$$

Finally, the augmented state-space model can be obtained by combining both (14) and (15), which results in

$$
\begin{aligned}
\mathbf{x}_{i}(k+1) & =\mathbf{A} \mathbf{x}_{i}(k)+\mathbf{B} \Delta u_{i}(k) \\
y_{i}(k) & =\mathbf{C} \mathbf{x}_{i}(k) .
\end{aligned}
$$

At this point, it is easy to find the matrices of the augmented model $\mathbf{A}, \mathbf{B}$, and $\mathbf{C}$ as a function of the matrices of the reduced model $\mathbf{A}_{m}, \mathbf{B}_{m}$, and $\mathbf{C}_{m}$, yielding:

$$
\begin{aligned}
\mathbf{A} & =\left[\begin{array}{cc}
\mathbf{A}_{m} & \mathbf{o}_{3}^{T} \\
\mathbf{C}_{m} \mathbf{A}_{m} & 1
\end{array}\right] \\
\mathbf{B} & =\left[\begin{array}{c}
\mathbf{B}_{m} \\
\mathbf{C}_{m} \mathbf{B}_{m}
\end{array}\right] \\
\mathbf{C} & =\left[\begin{array}{ll}
\mathbf{o}_{3} & 1
\end{array}\right]
\end{aligned}
$$

where $\mathbf{o}_{3}=\left[\begin{array}{lll}0 & 0 & 0\end{array}\right]$.

Note that since this model is an incremental model, the term $\boldsymbol{\eta}_{i}$ is removed from (7). Then, the effects of the model uncertainties and the steady-state values are eliminated, and also, a zero steady-state error can be achieved.

\section{B. Predictive control within a predictive horizon}

In this section, the model with an embedded integrator which has been previously presented is used to design the proposed MPC controller. In order to obtain the control signals, a prediction of the inverter-side currents is computed inside a predictive horizon whose dimension is $N_{p}$. The main objective of a CCS-MPC is to find a control signals vector of dimension $N_{p}$, where $N_{c} \leq N_{p}$, such that the error between the reference current and the prediction is minimum. Then, considering the sampling instant $k_{j}$, the vector of future incremental control actions can be expressed as follows:

$$
\Delta \mathbf{u}_{i}=\left[\begin{array}{llll}
\Delta u_{i}\left(k_{j}\right) & \Delta u_{i}\left(k_{j}+1\right) & \ldots & \Delta u_{i}\left(k_{j}+N_{c}-1\right)
\end{array}\right]
$$

With regard to the future state variables, they are expressed as:

$$
\mathbf{x}_{i}\left(k_{j}+1 \mid k_{j}\right), \mathbf{x}_{i}\left(k_{j}+2 \mid k_{j}\right), \ldots, \mathbf{x}_{i}\left(k_{j}+N_{p} \mid k_{j}\right)
$$


where $\mathbf{x}_{i}\left(k_{j}+m \mid k_{j}\right)$ is the prediction of the state variables at the sampling instant $k_{j}+m$ according to the values of $\mathbf{x}_{i}\left(k_{j}\right)$. Then, the predicted values can be computed as it is shown in [14]:

$$
\begin{aligned}
\mathbf{x}_{i}\left(k_{j}+1 \mid k_{j}\right) & =\mathbf{A} \mathbf{x}_{i}\left(k_{j}\right)+\mathbf{B} \Delta u_{i}\left(k_{j}\right) \\
\mathbf{x}_{i}\left(k_{j}+2 \mid k_{j}\right) & =\mathbf{A}^{2} \mathbf{x}_{i}\left(k_{j}\right)+\mathbf{A B} \Delta u_{i}\left(k_{j}\right)+\mathbf{B} \Delta u\left(k_{j}+1\right) \\
\mathbf{x}_{i}\left(k_{j}+N_{p} \mid k_{j}\right) & =\mathbf{A}^{N_{p}} \mathbf{x}_{i}\left(k_{j}\right)+\mathbf{A}^{N_{p}-1} \mathbf{B} \Delta u_{i}\left(k_{j}\right) \\
& +\mathbf{A}^{N_{p}-2} \mathbf{B} \Delta u_{i}\left(k_{j}+1\right)+\ldots \\
& \vdots \\
& +\mathbf{A}^{N_{p}-N_{c}} \mathbf{B} \Delta u_{i}\left(k_{j}+N_{c}-1\right)
\end{aligned}
$$

Now, the predicted outputs can be obtained from the predicted state variables in the same way:

$$
y_{i}\left(k_{j}+1 \mid k_{j}\right)=\mathbf{C A} \mathbf{x}_{i}\left(k_{j}\right)+\mathbf{C B} \Delta u_{i}\left(k_{j}\right)
$$

and through extrapolation, it gives:

$$
\begin{aligned}
y_{i}\left(k_{j}+N_{p} \mid k_{j}\right) & =\mathbf{C A}^{N_{p}} \mathbf{x}_{i}\left(k_{j}\right)+\mathbf{C A}^{N_{p}-1} \mathbf{B} \Delta u_{i}\left(k_{j}\right) \\
& +\mathbf{C A}^{N_{p}-2} \mathbf{B} \Delta u_{i}\left(k_{j}+1\right)+\ldots \\
& \vdots \\
& +\mathbf{C A}^{N_{p}-N_{c}} \mathbf{B} \Delta u_{i}\left(k_{j}+N_{c}-1\right)
\end{aligned}
$$

Finally, (27) and (28) can be rewritten in a matrix form as:

$$
\mathbf{y}_{i}=\mathbf{F}_{i} \mathbf{x}_{i}\left(k_{j}\right)+\mathbf{G}_{i} \Delta \mathbf{u}_{i}
$$

where $\mathbf{y}_{i}$ is a vector of dimension $N_{p}$ which contains the predicted outputs, and the matrices $\mathbf{F}_{i}$ and $\mathbf{G}_{i}$ are given by:

$$
\begin{aligned}
& \mathbf{F}_{i}=\left[\begin{array}{c}
\mathbf{C A} \\
\mathbf{C A}^{2} \\
\mathbf{C A}^{3} \\
\vdots \\
\mathbf{C A}^{N_{p}}
\end{array}\right] \\
& \mathbf{G}_{i}=\left[\begin{array}{ccccc}
\mathbf{C B} & 0 & 0 & \cdots & 0 \\
\mathbf{C A B} & \mathbf{C B} & 0 & \cdots & 0 \\
\mathbf{C A}^{2} \mathbf{B} & \mathbf{C A B} & \mathbf{C B} & \cdots & 0 \\
\cdot & \cdot & \cdot & \cdot & \cdot \\
\cdot & \cdot & \cdot & \cdot & \cdot \\
\mathbf{C A}^{\dot{N}_{p}-1} \mathbf{B} & \mathbf{C A}^{\dot{N}_{p}-2} \mathbf{B} & \cdots & \cdots & \mathbf{C A}^{N_{p}-N_{c}} \mathbf{B}
\end{array}\right]
\end{aligned}
$$

\section{State estimation}

In order to obtain the state space vector defined in (16), $\mathbf{x}_{i}(k)=\left[\begin{array}{ll}\Delta \mathbf{x}_{m, i}(k) & y_{i}(k)\end{array}\right]^{T}$, first $\mathbf{x}_{m, i}(k)$ is estimated. According to (12), this vector contains the state variables of the system. In this paper, the state variables for both $\alpha$ and $\beta$ channels have been estimated by using a KF as follows:

$$
\begin{aligned}
\hat{\mathbf{x}}_{m, i}(k+1) & =\mathbf{A}_{m} \hat{\mathbf{x}}_{m, i}(k)+\mathbf{B}_{m} u_{i}(k) \\
& +\mathbf{L}_{o b s}\left(i_{1 i}-\mathbf{C}_{m} \hat{\mathbf{x}}_{m, i}(k)\right)
\end{aligned}
$$

where $\mathbf{L}_{o b s}$ is the observer gain, which has been adjusted according to the Kalman filter algorithm [8].

\section{Cost function minimization}

The main objective of an MPC technique is to minimize the error between the predicted output and the reference. By assuming that the reference signal is maintained practically constant inside the predictive window, the following reference current vector can be defined:

$$
\mathbf{i}_{i}^{*}=\left[\begin{array}{llll}
1 & 1 & \ldots & 1
\end{array}\right]^{T} i_{i}^{*}\left(k_{j}\right)=\overline{\mathbf{r}} i_{i}^{*}\left(k_{j}\right)
$$

where $i_{i}^{*}\left(k_{j}\right)$ is the reference current at the sampling instant $k_{j}$ and $\overline{\mathbf{r}}$ is a column vector of ones that has the same size as the prediction horizon $N_{p}$.

The main objective of the control algorithm is to obtain the optimum control signals vector, $\Delta u_{i}$, so that the error between the reference current and the predicted output is minimum. To this end, the following cost functions for both $\alpha$ and $\beta$ can be defined [14]:

$$
\mathbf{J}_{i}=\left(\mathbf{i}_{i}^{*}-\mathbf{y}_{i}\right)^{T}\left(\mathbf{i}_{i}^{*}-\mathbf{y}_{i}\right)+\Delta \mathbf{u}_{i}^{T} \mathbf{R} \Delta \mathbf{u}_{i}
$$

where $\mathbf{R}=r_{\omega} \mathbf{I}_{N_{c} \times N_{c}}$ is a diagonal matrix and $r_{\omega}>0$ is the control effort used as a tuning parameter to adjust a desired closed loop performance. In order to minimize (34), equation (29) is used in (34). Then, as indicated in [14], by taking the derivative of $\mathbf{J}_{i}$ with respect to $\Delta \mathbf{u}_{i}$ we obtain:

$$
\frac{\partial \mathbf{J}_{i}}{\partial \Delta \mathbf{u}_{i}}=-2 \mathbf{G}_{i}^{T}\left(\mathbf{i}_{i}^{*}-\mathbf{F}_{i} \mathbf{x}_{i}\left(k_{j}\right)\right)+2\left(\mathbf{G}_{i}^{T} \mathbf{G}_{i}+\mathbf{R}\right) \Delta \mathbf{u}_{i}
$$

By equalizing (35) to zero and taking into account (33) the optimal incremental control signal vector for both $\alpha$ and $\beta$ channels, the following expression for the incremental control signals is obtained:

$$
\Delta \mathbf{u}_{i}\left(k_{j}\right)=\left(\mathbf{G}_{i}^{T} \mathbf{G}_{i}+\mathbf{R}\right)^{-1} \mathbf{G}_{i}^{T}\left(\overline{\mathbf{r}} i_{i}^{*}\left(k_{j}\right)-\mathbf{F}_{i} \mathbf{x}_{i}\left(k_{j}\right)\right)
$$

Note that the vector $\Delta \mathbf{u}_{i}$ contains all the incremental control signal values from the sampling instant $k_{j}$ to $k_{j}+N_{c}-1$. Since a receding horizon control is used, only the first sample of this vector is considered to generate the control signal. Then, according to the definition in (22), the actual incremental control signal can be expressed as follows

$$
\Delta u_{i}\left(k_{j}\right)=\mathbf{W}\left(\mathbf{G}_{i}^{T} \mathbf{G}_{i}+\mathbf{R}\right)^{-1} \mathbf{G}_{i}^{T}\left(\overline{\mathbf{r}} i_{i}^{*}\left(k_{j}\right)-\mathbf{F}_{i} \mathbf{x}_{i}\left(k_{j}\right)\right)
$$

where $\mathbf{W}=\left[\begin{array}{lllll}1 & 0 & 0 & \ldots & 0\end{array}\right]$ whose dimension is $N_{c}$.

Finally, the last step to obtain the optimum control signal is to add the incremental value, $\Delta u_{i}\left(k_{j}\right)$, to the control signal value in the previous sampling instant, $u_{i}\left(k_{j}-1\right)$. Then, one has:

$$
u_{i}\left(k_{j}\right)=\Delta u_{i}\left(k_{j}\right)+u_{i}\left(k_{j}-1\right)
$$

\section{E. Closed loop control system}

In this section, the closed-loop system is analyzed. In the last subsection, it has been shown that the optimum incremental control signal value at the sampling instant $k_{j}$ is defined by equation (37). By paying attention to this expression, it is found that at a given $k_{j}$ the optimal control vector $\Delta u_{i}$ can be separated into two parts. The first part has a dependence on the reference current while the second part corresponds to a classic case of a state feedback control. 
Since (17)-(18) is a time-invariant system, the incremental control defined by (37) can be expressed in terms of a state feedback controller as follows

$$
\Delta u_{i}(k)=K_{r} i_{i}^{*}(k)-\mathbf{K}_{c} \mathbf{x}_{i}(k)
$$

where

$$
\begin{aligned}
& K_{r}=\mathbf{W}\left(\mathbf{G}_{i}^{T} \mathbf{G}_{i}+\mathbf{R}\right)^{-1} \mathbf{G}_{i}^{T} \overline{\mathbf{r}} \\
& \mathbf{K}_{c}=\mathbf{W}\left(\mathbf{G}_{i}^{T} \mathbf{G}_{i}+\mathbf{R}\right)^{-1} \mathbf{G}_{i}^{T} \mathbf{F}_{i}
\end{aligned}
$$

In the aforementioned expressions, $K_{r}$ is a scalar while $\mathbf{K}_{c}$ is a vector which matches with the dimension of $\mathbf{x}_{i}$.

Now, to prove the effect of the predictive control in the system dynamics, (39) is replaced in (17):

$$
\mathbf{x}_{i}(k+1)=\mathbf{A} \mathbf{x}_{i}(k)-\mathbf{B} \mathbf{K}_{c} \mathbf{x}(k)-K_{r} \mathbf{B} i_{i}^{*}(k)
$$

or equivalently

$$
\mathbf{x}_{i}(k+1)=\left(\mathbf{A}-\mathbf{B} \mathbf{K}_{c}\right) \mathbf{x}_{i}(k)-K_{r} \mathbf{B} i_{i}^{*}(k)
$$

where the closed loop eigenvalues can be obtained by solving the determinant

$$
\left|A-\mathbf{B K}_{c}-\lambda \mathbf{I}\right|=0
$$

The eigenvalues have a dependence of $\mathbf{K}_{c}$ and as a consequence, of $\mathbf{R}$. As evidenced by the last expression, by selecting the appropriate value of $\mathbf{R}$ in (37), a desired dynamics of the MPC can be obtained. In Table $I$, the control effort $r_{\omega}$ is chosen according to (44) in order to obtain a fast transient response as it will shown in the experimental results section.

\section{HARMONICS COMPENSATION}

In the case of grid harmonics, the three-phase currents can be distorted. The proposed controller is addressed to reduce its effect. To this end, a feedforward term is introduced. To prove this, equation (4) is discretized as follows:

$$
\left(L_{1 o}+L_{2 o}\right) \frac{\mathbf{i}_{1 \alpha \beta}(k+1)-\mathbf{i}_{1 \alpha \beta}(k)}{T_{s}}=\frac{V_{d c o}}{2} \mathbf{d}_{\alpha \beta}-\mathbf{v}_{\alpha \beta}
$$

According to the control scheme presented in Fig.2 and taking into account (38), the control signal applied to the converter is obtained as:

$$
\mathbf{d}_{\alpha \beta}\left(k_{j}\right)=\Delta \mathbf{u}_{\alpha \beta}\left(k_{j}\right)+\mathbf{u}_{\alpha \beta}\left(k_{j}-1\right)+\frac{2}{V_{d c}} \mathbf{v}_{\alpha \beta}\left(k_{j}\right)
$$

where $\frac{2}{V_{d c}} \mathbf{v}_{\alpha \beta}$ is a feedforward term.

By using (46) in (45), one has:

$$
\begin{aligned}
\mathbf{i}_{1 \alpha \beta}(k+1) & =\mathbf{i}_{1 \alpha \beta}(k)+\frac{V_{d c o} T_{s}}{2\left(L_{1 o}+L_{2 o}\right)}\left(\Delta \mathbf{u}_{\alpha \beta}\left(k_{j}\right)\right. \\
& \left.+\mathbf{u}_{\alpha \beta}\left(k_{j}-1\right)\right)
\end{aligned}
$$

The aforementioned equation shows that the inverter-side currents discrete expression does not depend on the PCC voltages, and as a consequence, not on the grid harmonics either. With this method, it is not necessary to use any harmonics model [23].

\section{SEQUENCE EXTRACTOR AND REFERENCE CURRENT GENERATOR}

The proposed controller is designed to track only the PCC voltage positive sequence. In fact, in the case of voltage sags, the positive and negative sequences can be obtained without using any particular sequence extractor. Taking advantage of the proposed model, where the direct and quadrature PCC voltages are available, the positive sequence can be obtained as it is described below.

Using the Clarke transformation:

$$
\left[T_{\alpha \beta}\right]=\frac{2}{3}\left[\begin{array}{ccc}
1 & -\frac{1}{2} & -\frac{1}{2} \\
0 & \frac{\sqrt{3}}{2} & -\frac{\sqrt{3}}{2}
\end{array}\right]
$$

and applying this transformation to the estimated PCC voltages and their quadratures, one has:

$$
\begin{gathered}
{\left[\begin{array}{c}
\hat{v}_{\alpha} \\
\hat{v}_{\beta}
\end{array}\right]=\left[T_{\alpha \beta}\right]\left[\begin{array}{l}
\hat{v}_{a} \\
\hat{v}_{b} \\
\hat{v}_{c}
\end{array}\right]} \\
{\left[\begin{array}{c}
\hat{v}_{\alpha}^{\perp} \\
\hat{v}_{\beta}^{\perp}
\end{array}\right]=\left[T_{\alpha \beta}\right]\left[\begin{array}{c}
\hat{v}_{a}^{\perp} \\
\hat{v}_{b}^{\perp} \\
\hat{v}_{c}^{\perp}
\end{array}\right] .}
\end{gathered}
$$

From the last expressions, the estimated positive sequence, $\hat{v}_{\alpha}^{+}$ and $\hat{v}_{\beta}^{+}$can be obtained as follows:

$$
\begin{aligned}
& \hat{v}_{\alpha}^{+}=\frac{1}{2} \hat{v}_{\alpha}+\frac{1}{2} \hat{v}_{\beta}^{\perp} \\
& \hat{v}_{\beta}^{+}=-\frac{1}{2} \hat{v}_{\alpha}^{\perp}+\frac{1}{2} \hat{v}_{\beta} .
\end{aligned}
$$

With this solution, the reference currents can be generated using only the positive sequence of the PCC voltages:

$$
\begin{aligned}
i_{\alpha}^{*} & =k_{p} \hat{v}_{\alpha}^{+}+k_{q} \hat{v}_{\beta}^{+} \\
i_{\beta}^{*} & =k_{p} \hat{v}_{\beta}^{+}-k_{q} \hat{v}_{\alpha}^{+}
\end{aligned}
$$

where the value of parameters $k_{p}$ and $k_{q}$ are expressed as [24]:

$$
\begin{aligned}
& k_{p}=\frac{2 P^{*}}{3\left(\left(\hat{v}_{\alpha}^{+}\right)^{2}+\left(\hat{v}_{\beta}^{+}\right)^{2}\right)} \\
& k_{q}=\frac{2 Q^{*}}{3\left(\left(\hat{v}_{\alpha}^{+}\right)^{2}+\left(\hat{v}_{\beta}^{+}\right)^{2}\right)}
\end{aligned}
$$

being $P^{*}$ and $Q^{*}$ the active and reactive reference powers, respectively.

\section{CONTROLLER IMPLEMENTATION}

This section deals with the implementation of the proposed MPC. Fig.3 and Fig.4 show a block diagram and a pseudocode of the control algorithm, respectively.

Fig.3 shows a simplified block diagram of the MPC algorithm for the $\alpha$-channel. The inputs to this block are the inverter-side current, its reference and the voltage at the PCC. A KF estimator (32) is used to generate the estimated vector $\hat{\mathbf{x}}_{m \alpha}(k)$. This vector allows us to obtain the new space state vector $\mathbf{x}_{\alpha}(k)$, formed by $\Delta \mathbf{x}_{m, \alpha}(k)$ and the output $y_{\alpha}(k)$. A cost function minimization (37) is used to obtain the optimum control signal $\Delta u_{\alpha}(k)$. Finally, $u_{\alpha}(k)$ is added to 


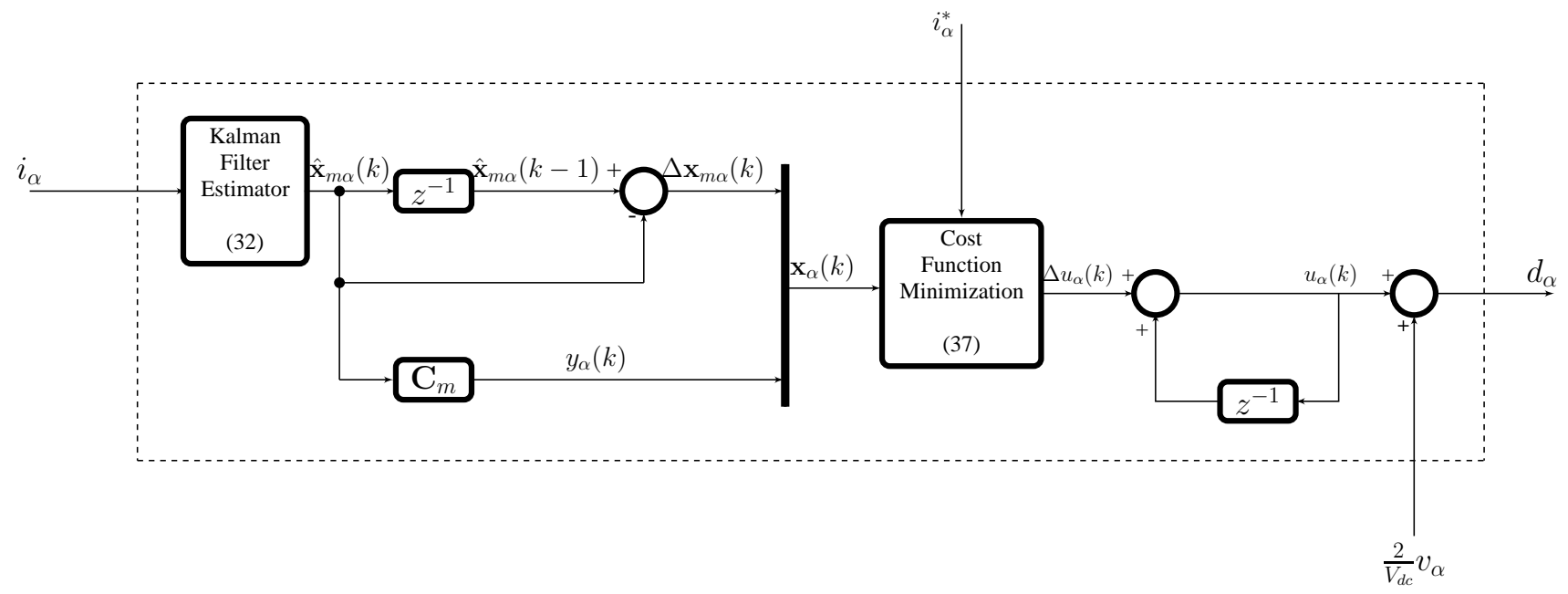

Fig. 3. Block diagram for $\alpha$-channel of the proposed MPC

TABLE I

\section{SYSTEM PARAMETERS}

\begin{tabular}{lcc}
\hline \hline Description & Symbol & Value \\
\hline Grid voltage & $V_{g r i d}$ & $110 V_{r m s}$ \\
Grid frequency & $f_{o}$ & $60 \mathrm{~Hz}$ \\
Nominal dc-link voltage & $V_{d c o}$ & $400 \mathrm{~V}$ \\
Nominal filter input inductance & $L_{1 o}$ & $5 \mathrm{mH}$ \\
Nominal filter capacitor & $C_{o}$ & $2.2 \mathrm{uF}$ \\
Nominal filter output inductance & $L_{2 o}$ & $2 \mathrm{mH}$ \\
Grid inductance & $L_{g}$ & $0.5 \mathrm{mH}$ \\
Sampling frequency & $f_{s}$ & $10 \mathrm{kHz}$ \\
Switching frequency & $f_{s w}$ & $10 \mathrm{kHz}$ \\
Prediction horizon & $N_{p}$ & 8 \\
Control horizon & $N_{c}$ & 4 \\
Control effort & $r_{\omega}$ & 2 \\
\hline
\end{tabular}

a feedforward term $\frac{V_{d c}}{2} v_{\alpha}$ in order to generate the control signal $d_{\alpha}$.

Fig. 4 shows the pseudo-code for the controller implementation. The subindex $i$ represents $\alpha$ or $\beta$ indistinctly. The optimization problem can be solved offline as it happens to an infinite-horizon or linear quadratic regulator (LQR). For this reason, all the matrices and gains for the MPC are computed offline with customized Matlab functions. Besides, since a receding horizon is used, only the first column of the gains $K_{r}$ and $\mathbf{K}_{c}$ is computed. Once these gains are obtained, the algorithm is executed as shown in the pseudo-code.

\section{A. Selection of the control parameters}

The control effort parameter, $r_{\omega}$ has been adjusted according to the desired position of the closed-loop eigenvalues (44). On the other hand, as it has previously been stated, the prediction and control horizons $\left(N_{p}\right.$ and $N_{c}$ ) are adjusted to ensure stability. Several simulations have been performed, which has been used to determine the adequate values of the control algorithm parameters. Besides, from these simulations, pole maps has been obtained.

Fig.5 shows the position of the closed loop poles in two different scenarios for a control effort variation in the range $0 \leq r_{\omega} \leq 40$. Note that the poles in red are the closedloop poles for $r_{\omega}=2$ which is the selected value used in the experimental results.

In Fig.5(a), the control and prediction horizons are selected as $N_{c}=1$ and $N_{p}=2$. For these horizons, the poles are unstable for a wide range of $r_{\omega}$. Conversely, in Fig.5(b) the control and prediction horizons are increased to $N_{c}=4$ and $N_{p}=8$. As it can be seen, the stability is ensured for all values of the control effort. Then, $\mathrm{Np}=8$, and $\mathrm{Nc}=4$ are used for the controller implementation. Notice that as the prediction horizon is increased, the system tends to an unstable system, as shown in Fig.5(b). Besides, an increment in the prediction horizon makes the control action less aggressive which results in a slower transient response. The selected control and prediction horizons combined with a control effort $r_{\omega}=2$ leads to a stable system with a fast transient response, as verified experimentally in section VIII.

\section{EXPERIMENTAL VALIDATION}

In order to evaluate and compare the performances of the proposed control method, an experimental three-phase three-wire inverter prototype has been built using a 4.5kVA SEMIKRON full-bridge as the power converter. The TMS320F28M36 floating-point digital signal processor (DSP) has been chosen as the control platform with a sampling frequency of $10 \mathrm{kHz}$. The grid and the DC-link voltages have been generated using a PACIFIC 360-AMX and AMREL SPS1000-10-K0E3 source, respectively. The system parameters are listed in Table I. A photograph of the experimental setup is shown in Fig.6.

\section{A. Transient response}

The transient response in the case of a sudden step change in the reference current is analyzed in Fig.7. The figure shows, 
TASK : Controller $(h)$

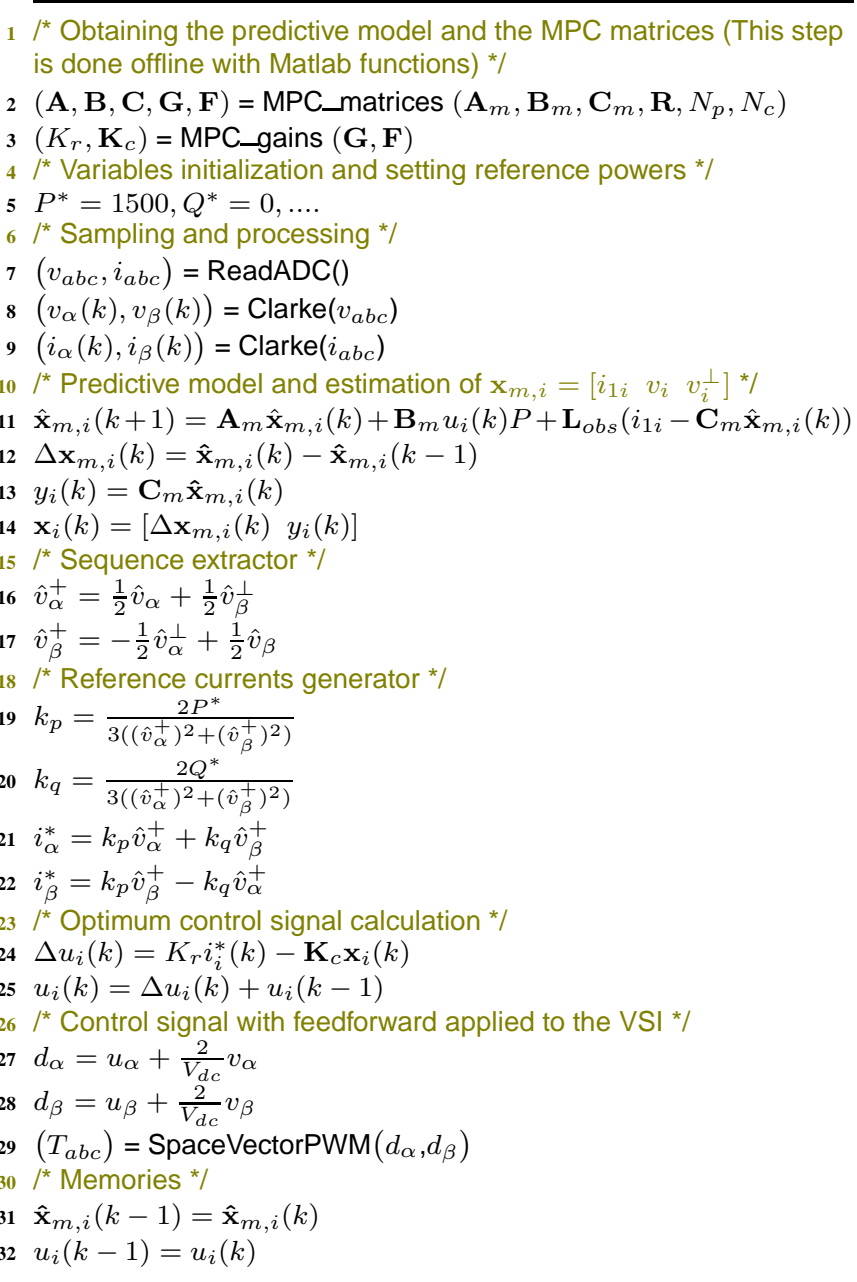

Fig. 4. Pseudo-code of the controller task.

from top to bottom, the PCC voltages and the three-phase grid currents, respectively. In this test, the reference current is changed from $1 \mathrm{~A}$ to $9.5 \mathrm{~A}$. As it is shown, a fast transient response is achieved. In addition, it can be seen that the controller can operate in a wide margin of the reference currents amplitude values without changing any parameter in the control algorithm.

Fig. 8 shows the transient response, but in this case, when an active or reactive power step change is carried out. First, in Fig.8(a), an active power step change from $0 \mathrm{~W}$ to $2000 \mathrm{~W}$ is performed while maintaining the reactive power reference to 0 $\operatorname{VAr}$ (see equations (55)-(56)). As it can be seen, the transient response is very fast even in the case of an important reference change. Besides, the reactive power is maintained to 0VAr, as expected.

Finally, Fig.8(b) shows the transient response but in this case, in the reactive power. For this test the reactive power reference has been changed from 0 VAr to $2000 \mathrm{VAr}$, while maintaining the active power to $0 \mathrm{~W}$. The figure shows also a fast transient response in the case of a sudden variation in the reactive power.

Note that in both tests, it is revealed that readjusting the

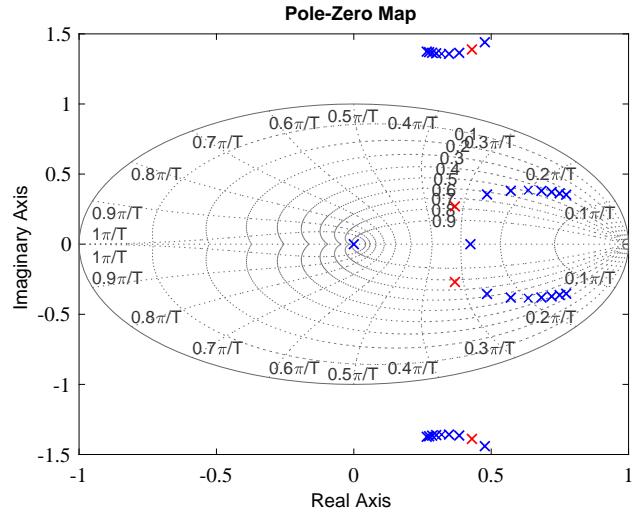

(a)

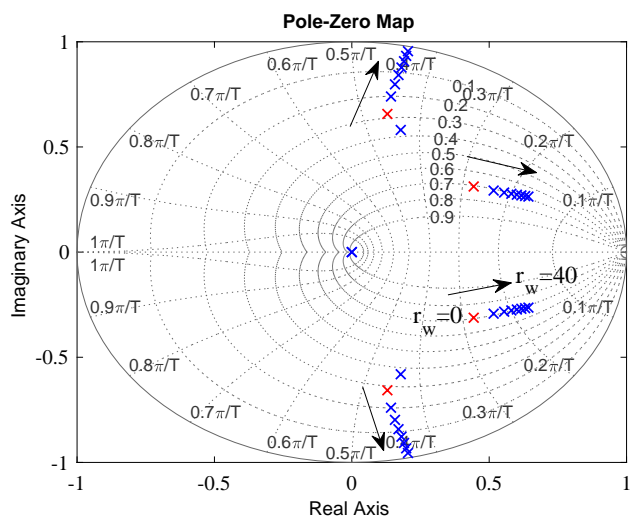

(b)

Fig. 5. Closed loop poles for $r_{\omega}$ variation from 0 to 40 . In red the poles for $r_{\omega}=2$, for the control and prediction horizons: a) $N_{c}=1, N_{p}=2$ and b) $N_{c}=4, N_{p}=8$.

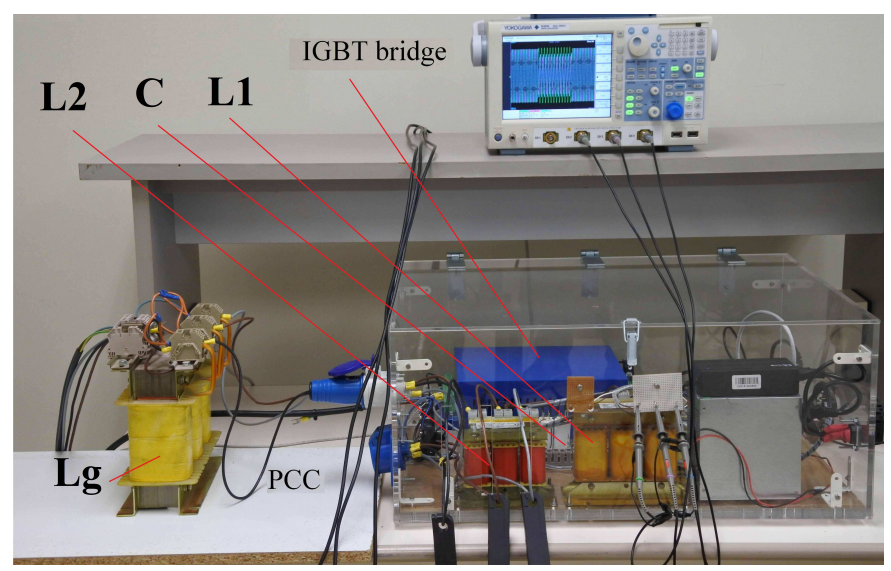

Fig. 6. Photograph of the experimental setup 


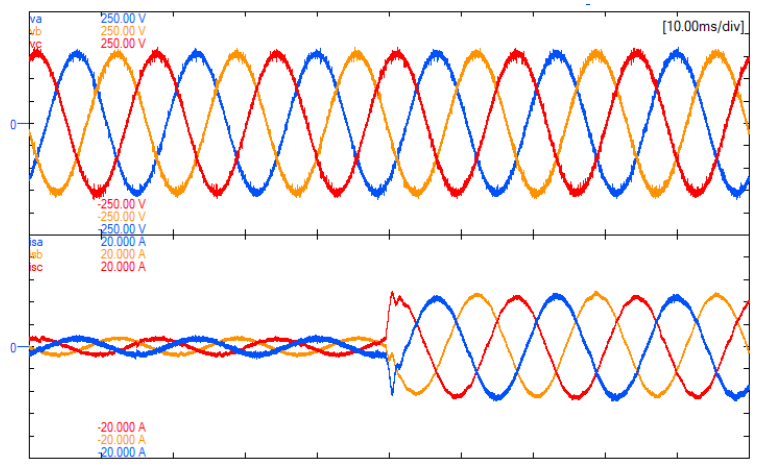

Fig. 7. From top to bottom: experimental PCC voltages (50V/div) and grid currents $(4 \mathrm{~A} / \mathrm{div})$ when a current reference step change from $1 \mathrm{~A}$ to $9.5 \mathrm{~A}$ is done.

controller parameters when the operating point is changed is not required.

\section{B. Controller performances in the case of grid harmonics}

In Fig.9, the controller performances in the case of grid harmonics are shown. Fig.9(a) shows, from top to bottom, the grid voltages with a THD of $14 \%$ (standard IEC-77A) and the grid currents when the feedforward term (see eq.(46)) is not introduced in the controller. As it can be seen, harmonics in the grid voltage deteriorate the quality of the currents injected into the grid. Conversely, Fig.9(b) shows the controller performances when the feedforward term is used. As shown in the figure, the quality of the grid currents is improved, providing a practically sinusoidal three-phase currents.

\section{Grid voltage unbalance}

The proposed controller has been tested under grid voltage unbalance. Fig.10 shows the performance of the controller under a grid voltage sag. This sag is characterized by a positive and negative sequence of $V^{+}=0.7$ p.u. and $V^{-}=0.3$ p.u. respectively, and with a phase angle between sequences of $\phi=-\pi / 6$

In this test, the currents are forced to track only the positive sequence of the PCC voltage. For this reason, the reference currents are obtained using the positive sequences of those voltages which are expressed in (53)-(54). The active power has been set to a reference value of $P^{*}=1500 \mathrm{~W}$. Note that the current amplitude is balanced and is increased during the sag in order to maintain the desired active power.

\section{Grid inductance variation}

In order to show the system behavior when the grid inductance varies, the following figure is presented. Fig.11 shows the experimental PCC voltages and grid currents when the grid inductance is changed from the nominal value $L_{g}=0.5 \mathrm{mH}$ to $L_{g}=5 \mathrm{mH}$. As it can be seen, the grid voltages are distorted and high switching noise can be observed. Moreover, since the reference currents are generated with the estimated PCC voltages obtained from the $\mathrm{KF}$, the three-phase grid currents are sinusoidal with a low ripple. Note that a step change is produced in order to test the controller in this situation.

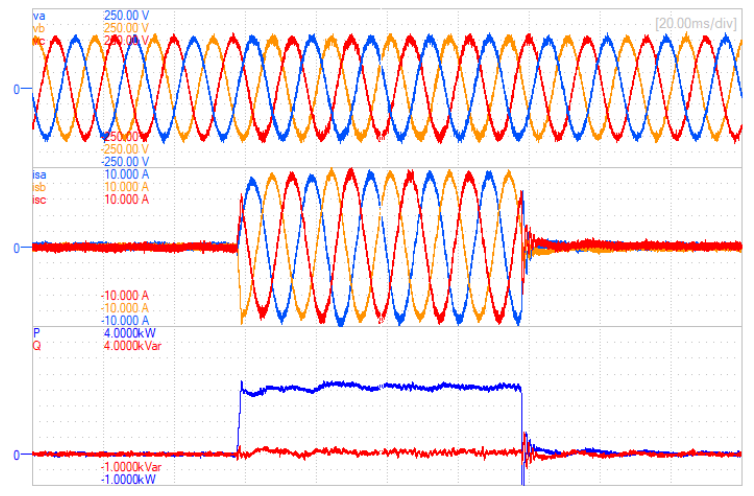

(a)

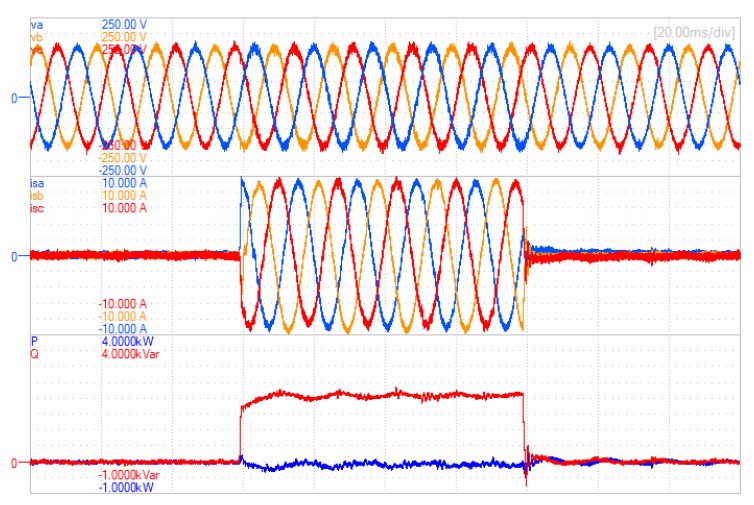

(b)

Fig. 8. From top to bottom: experimental PCC voltages $(50 \mathrm{~V} / \mathrm{div})$ grid currents $(2 \mathrm{~A} / \mathrm{div})$ and (a) an active power (500 W/div) step change from 0 to $2000 \mathrm{~W}$ and (b) a reactive power (500 VAr/div) step change from 0 to 2000 VAr.

\section{COMPARATIVE ANALYSIS}

This section deals with a comparative analysis between the FCS-MPC and the proposed controller. This comparison is in terms of switching frequency, THD, and computational load (CL), obtained according to the following expression:

$$
C L(\%)=f_{s} \times t_{e x} \times 100
$$

where $t_{e x}$ is the execution time.

In order to obtain the execution time of the algorithm, a DSP timer is used to measure the time of the controller task. For the THD calculation, the signals are also obtained with Matlab and a fast Fourier transform (FFT) is applied.

Besides, for this comparison, it is considered the distorted grid voltages used in the experimental results with a THD of $14 \%$.

In order to minimize the computational load, the optimization problem is solved offline, in a similar way to which it is done with an infinite-horizon or linear quadratic regulator (LQR). Moreover, the use of this reduced model helps to diminish the execution time even more, since the number of state variables decrease. Note that the reduction of the sampling frequency reduces the percentage of CL.

Table II shows three different strategies, the FCS-MPC, and the proposed controller, with and without a feedforward term.

In order to validate experimentally the FCS-MPC, the following cost function has been considered [13]: 


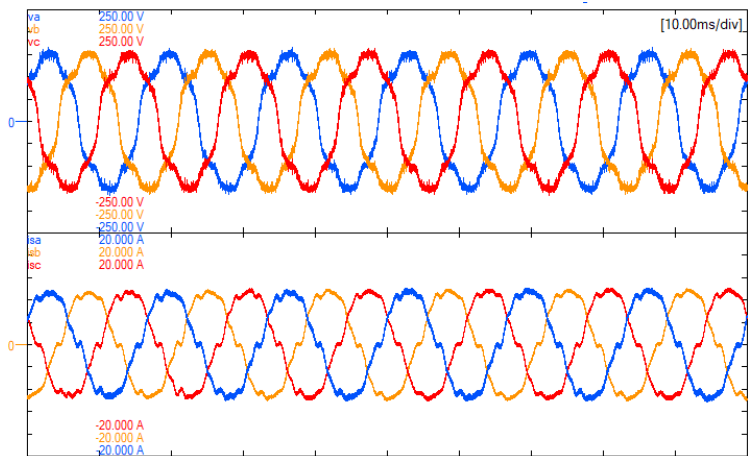

(a)

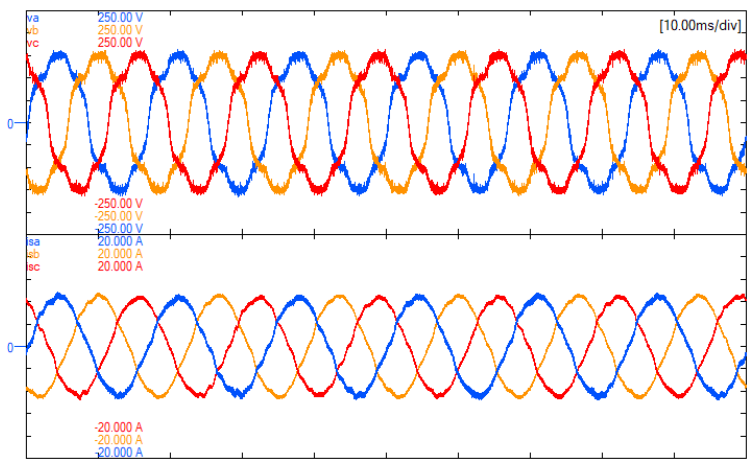

(b)

Fig. 9. From top to bottom: experimental PCC voltages (50V/div) and grid currents (4A/div) when (a) a feedforfard term is not used in the controller and (b) a feedforward term is used in the controller.

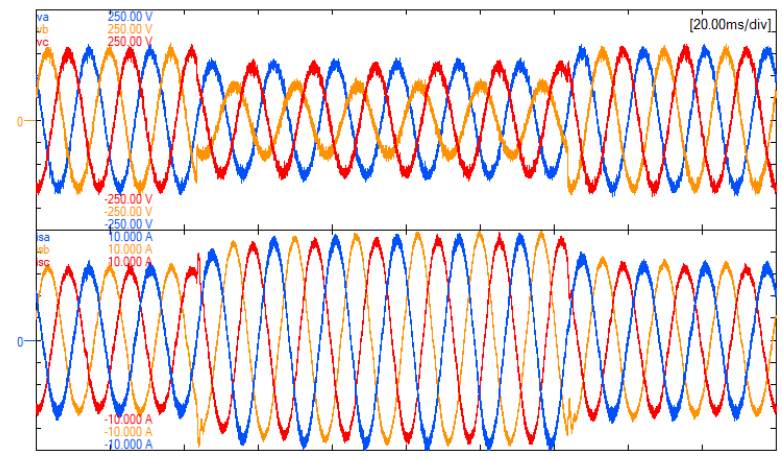

Fig. 10. From top to bottom: experimental PCC voltages (50V/div) and grid currents (2A/div) during a voltage sag.

$$
J=\left|i_{1 \alpha}^{*}-i_{1 \alpha}\right|+\left|i_{1 \beta}^{*}-i_{1 \beta}\right|
$$

By using (58), the FCS-MPC does not force a commutation in each sample period. Hence, the average switching frequency is variable, which leads to a spread current spectrum. This fact deteriorates the THD of grid currents in comparison with the CCS-MPC

Conversely, the proposed controller can operate at a fixed switching frequency without using any additional controllers, such as PI or PRES controllers. Note that the introduction of the feedforward term does not increase the algorithm complexity and has the advantage of reducing the THD.

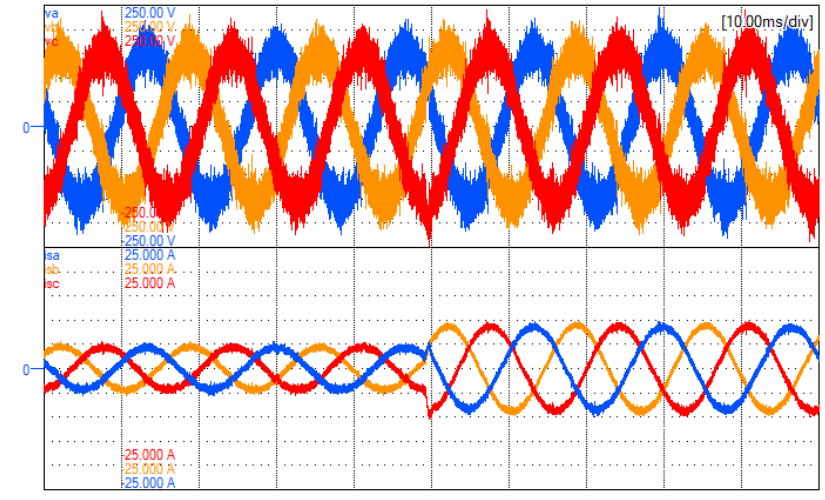

Fig. 11. From top to bottom: experimental PCC voltages (50V/div) and grid currents (5A/div) when a current reference step change from $1 \mathrm{~A}$ to $9.5 \mathrm{~A}$ is carried out.

TABLE II

COMPARATIVE ANALYSIS

\begin{tabular}{ccccc}
\hline \hline Algorithm & $f_{s}$ & $f_{s w}$ & THD & $C L$ \\
\hline FCS-MPC using KF & $40 \mathrm{KHz}$ & variable $(3 \mathrm{kHz}-7 \mathrm{kHz})$ & $4.2 \%$ & $82 \%$ \\
Proposal without FF & $10 \mathrm{kHz}$ & $10 \mathrm{kHz}$ & $10.2 \%$ & $31 \%$ \\
Proposal with FF & $10 \mathrm{kHz}$ & $10 \mathrm{kHz}$ & $3.1 \%$ & $31 \%$ \\
\hline
\end{tabular}

\section{CONClusions}

In this paper, a CCS-MPC for a three-phase voltage source inverter with an LCL filter has been presented. The proposed controller uses a reduced model with an embedded integrator in order to allow active damping while eliminating the model uncertainties and also, to achieve a zero steady state error. Besides, a reduction of the computational burden is also achieved. Simulation and experimental results show that this proposal does not require the use of PRES or PI controllers and a fixed switching frequency can be obtained. Finally, the use of a feedforward term added to the control signal can reduce the effect of grid voltage harmonics in three-phase currents. Experimental results have also shown that this proposal works correctly even in the case of grid harmonics and voltage sags.

\section{REFERENCES}

[1] K. Moslehi and R. Kumar, "A reliability perspective of the smart grid," IEEE Trans. Smart Grid, vol. 1, no. 1, pp. 57-64, June 2010.

[2] A. Ipakchi and F. Albuyeh, "Grid of the future," IEEE Power and Energy Magazine, vol. 7, no. 2, pp. 52-62, March 2009.

[3] S. Yang, Q. Lei, F. Z. Peng, and Z. Qian, "A robust control scheme for grid-connected voltage-source inverters," IEEE Trans. Ind. Electron., vol. 58, no. 1, pp. 202-212, Jan 2011.

[4] T. C. Y. Wang, Z. Ye, G. Sinha, and X. Yuan, "Output filter design for a grid-interconnected three-phase inverter," in Power Electronics Specialist Conference, 2003. PESC '03. 2003 IEEE 34th Annual, vol. 2, June 2003, pp. 779-784 vol.2.

[5] J. Xu, S. Xie, and T. Tang, "Active damping-based control for gridconnected lcl -filtered inverter with injected grid current feedback only," IEEE Trans. Ind. Electron., vol. 61, no. 9, pp. 4746-4758, Sept 2014.

[6] R. Guzman, L. G. de Vicua, J. Morales, M. Castilla, and J. Miret, "Model-based active damping control for three-phase voltage source inverters with LCL filter," IEEE Transactions on Power Electronics, vol. 32, no. 7, pp. 5637-5650, July 2017. 
[7] R. Guzman, L. G. de Vicua, M. Castilla, J. Miret, and J. de la Hoz, "Variable structure control for three-phase LCL-filtered inverters using a reduced converter model," IEEE Trans. Ind. Electron., vol. 65, no. 1, pp. 5-15, Jan 2018.

[8] R. Guzman, L. G. de Vicua, M. Castilla, J. Miret, and H. Martn, "Variable structure control in natural frame for three-phase grid-connected inverters with LCL filter," IEEE Trans. Power Electron., in press.

[9] P. Kanjiya, V. Khadkikar, and H. H. Zeineldin, "Optimal control of shunt active power filter to meet ieee std. 519 current harmonic constraints under nonideal supply condition," IEEE Trans. on Ind. Electron., vol. 62, no. 2, pp. 724-734, Feb 2015.

[10] L. Tarisciotti, A. Formentini, A. Gaeta, M. Degano, P. Zanchetta, R. Rabbeni, and M. Pucci, "Model predictive control for shunt active filters with fixed switching frequency," IEEE Trans. on Ind. Appl., vol. 53, no. 1, pp. 296-304, Jan 2017.

[11] T. H. Nguyen and K.-H. Kim, "Finite control set model predictive control with modulation to mitigate harmonic component in output current for a grid-connected inverter under distorted grid conditions," Energies, vol. 10, p. 907, Feb 2017.

[12] J. Hu and K. W. E. Cheng, "Predictive control of power electronics converters in renewable energy systems," Energies, vol. 10, p. 515, Feb 2017.

[13] S. Kouro, P. Cortes, R. Vargas, U. Ammann, and J. Rodriguez, "Model predictive control-a simple and powerful method to control power converters," IEEE Trans. Ind. Electron., vol. 56, no. 6, pp. 1826-1838, June 2009.

[14] L. Wang, Model Predictive Control System Design and Implementation Using MATLAB. Springer, 2009

[15] M. Trabelsi, S. Bayhan, K. A. Ghazi, H. Abu-Rub, and L. Ben-Brahim, "Finite-control-set model predictive control for grid-connected packedU-cells multilevel inverter," IEEE Trans. on Ind. Electron., vol. 63, no. 11 , pp. 7286-7295, Nov 2016.

[16] P. Kou, D. Liang, J. Li, L. Gao, and Q. Ze, "Finite-control-set model predictive control for dfig wind turbines," IEEE Trans. on Automation Science and Engineering, in press.

[17] C. Qi, X. Chen, P. Tu, and P. Wang, "Cell-by-cell-based finite-control-set model predictive control for a single-phase cascaded h-bridge rectifier," IEEE Transactions on Power Electronics, in press.

[18] M. Ramzi, H. Youlal, and M. Haloua, "State space model predictive control of an aerothermic process with actuators constraints," Intelligent Control and Automation, vol. 3, no. 1, pp. 50-58, Feb 2012.

[19] H. T. Nguyen, E. K. Kim, I. P. Kim, H. H. Choi, and J. W. Jung, "Model predictive control with modulated optimal vector for a threephase inverter with an lc filter," IEEE Trans. on Power Electron., vol. 33, no. 3, pp. 2690-2703, March 2018.

[20] M. G. Judewicz, S. A. Gonzlez, J. R. Fischer, J. F. Martnez, and D. O. Carrica, "Inverter-side current control of grid-connected voltage source inverters with LCL filter based on generalized predictive control," IEEE Journal of Emerging and Selected Topics in Power Electronics, in press.

[21] A. A. Ahmed, B. K. Koh, and Y. I. Lee, "A comparison of finite control set and continuous control set model predictive control schemes for speed control of induction motors," IEEE Trans. on Ind. Inform., vol. 14, no. 4, pp. 1334-1346, April 2018

[22] S. Golestan, E. Ebrahimzadeh, J. M. Guerrero, and J. C. Vasquez, "An adaptive resonant regulator for single-phase grid-tied vscs," IEEE Trans. Power Electron., vol. 33, no. 3, pp. 1867-1873, March 2018.

[23] J. Kanieski, R. Cardoso, H. Pinheiro, and H. Grundling, "Kalman filterbased control system for power quality conditioning devices," IEEE Trans. Ind. Electron., vol. 60, no. 11, pp. 5214-5227, Nov 2013.

[24] M. Castilla, J. Miret, A. Camacho, J. Matas, and L. G. de Vicua, "Voltage support control strategies for static synchronous compensators under unbalanced voltage sags," IEEE Trans. Ind. Electron., vol. 61, no. 2, pp. 808-820, Feb 2014.

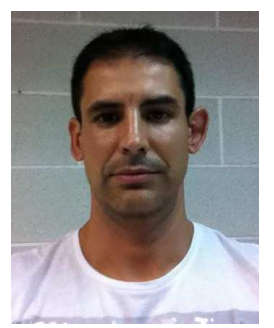

Ramon Guzman received the B.S., the M.S. and the Ph.D. degrees in telecommunications engineering from the Technical University of Catalonia, Barcelona, Spain, in 1999, 2004 and 2016 , respectively. He is currently an Associate Professor with the Department of Automatic Control in the Technical University of Catalonia. His research interests include nonlinear and adaptive control for three-phase power converters.

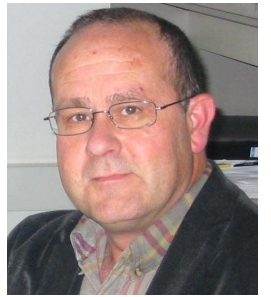

Luis Garcia de Vicuña a received the M.S. and Ph.D. degrees in telecommunication engineering from the Technical University of Catalonia, Barcelona, Spain, in 1980 and 1990, respectively, and the Ph.D. degree in electrical engineering from the Paul Sabatier University, Toulouse, France, in 1992. From 1980 to 1982 , he was an Engineer with a control applications company in Spain. He is currently a Full Professor with the Department of Electronic Engineering, Technical University of Catalonia, where he teaches courses on power electronics. His research interests include power electronics modeling, simulation and control, active power filtering, and high-power-factor ac/dc conversion.

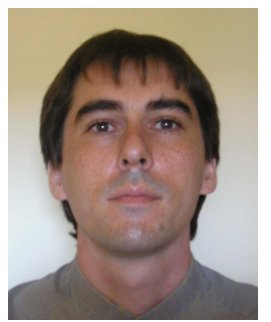

Antonio Camacho received the B.S. degree in chemical engineering, the M.S. degree in automation and industrial electronics, and the Ph.D. degree in electronic engineering, from the Technical University of Catalonia, Barcelona, Spain in 2000, 2009 and 2015 respectively. His research interests include networked and embedded control systems, industrial informatics, and power electronics.

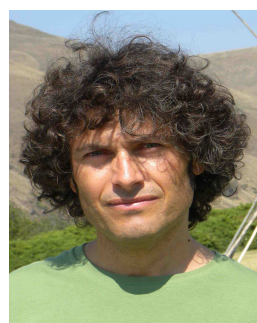

Jaume Miret (M98) received the B.S. degree in telecommunications, M.S. degree in electronics, and Ph.D. degree in electronics from the Universitat Politecnica de Catalunya, Barcelona, Spain, in 1992, 1999, and 2005, respectively. From 1993 to 2011, he was an Assistant Professor in the Department of Electronic Engineering, Universitat Politecnica de Catalunya, Spain. Since 2011 he has been an Associate Professor in the Universitat Politecnica de Catalunya. His active power filters, and digital control. research interests include dc-to-ac converters,

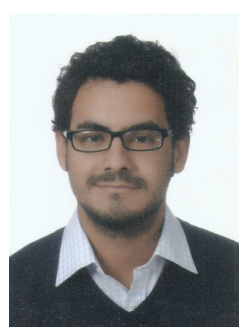

Juan M. Rey was born in Bucaramanga, Colombia in 1989. He received the B.S. in electrical engineering from Universidad Industrial de Santander, Bucaramanga, Colombia, in 2012. He is currently working toward the Ph.D. degree in the Department of Electronic Engineering, Technical University of Catalonia, Spain. Since 2013, he has been with the Electrical, Electronic and Telecommunications Engineering School (E3T), Universidad Industrial de Santander, Bucaramanga Colombia, where he is currently an Assistant Professor. His research interest are power electronics and control for distributed generation and microgrids. 